\title{
The Effect of Comprehensive Hand Repetitive Intensive Strength Training (CHRIST) Using Motion Analysis in Children with Cerebral Palsy
}

\author{
Dong-A Kim, M.D., Jung-Ah Lee, Ph.D. ${ }^{1}$, Pill-Woo Hwang, B.M. ${ }^{1}$, Min-Jin Lee, B.S. ${ }^{1}$, Hyun-Kyung Kim, M.D., \\ Jeong-Joon Park, M.D., Joshua H. You, Ph.D. ${ }^{2}$, Dong-Ryul Lee, M.Sc. ${ }^{2}$, Nam-Gi Lee, M.Sc. ${ }^{2}$ \\ Department of Rehabilitation Medicine, National Rehabilitation Hospital, \\ ${ }^{1}$ Department of Motor \& Cognitive Rehabilitation, Korea National Rehabilitation Research Institute, Seoul 142-884, \\ ${ }^{2}$ Department of Physical Therapy, Yonsei University, Wonju 220-710, Korea
}

\begin{abstract}
Objective To investigate the effect of Comprehensive Hand Repetitive Intensive Strength Training (CHRIST) on upper limb function in children with cerebral palsy using motion analysis.

Method The subjects in this study included 19 children ( 10 males, 9 females, mean age $=8.8$ years) with cerebral palsy. The experimental group $(\mathrm{n}=10)$ received CHRIST and general rehabilitation therapy. The control group $(\mathrm{n}=9)$ received a home program as well as general rehabilitation therapy. Both groups received30 sessions of CHRIST or home program training for 60 minutes per session 3 times a week during the 10-week period. The reaching movements were captured by a motion analysis system. Kinematic variables including movement time (MT), mean velocity (MV), normalized jerk score (NJS), mean angular velocity (MAV) and normalized jerk score of the shoulder, elbow and wrist joint with comfortable and fast speed were analyzed between groups and the pre-post training group.

Results After pre- and post-training experimental group, MT, MV, NJS, MAV of shoulder, elbow, wrist and NJS of elbow and wrist improved significantlyin reaching movement of both comfortable and fast speed $(\mathrm{p}<0.05)$. However, After pre- and post-training control group, MV improved significantlyin reaching movement of only comfortable speed $(\mathrm{p}<0.05)$. Between two groups, MT and MAV of the elbow at comfortable speed and NJS of the elbow at fast speed were statisticallysignificant $(\mathrm{p}<0.05)$.

Conclusion CHRIST proved to be an effective intervention for improving upper limb extremity function of reaching movement in children with cerebral palsy.
\end{abstract}

Key Words Cerebral palsy, Muscle strength, Upper extremity, Motion

Received August 22, 2011; Accepted October 24, 2011

Corresponding author: Jeong-Joon Park

Department of Rehabilitation Medicine, National Rehabilitation Hospital, 520, Suyu 5-dong, Gangbuk-gu, Seoul 142-884, Korea

Tel: +82-2-901-1700, Fax: +82-2-902-3835, E-mail: jjpark0324@gmail.com (c) This is an open-access article distributed under the terms of the Creative Commons Attribution Non-Commercial License (http:// creativecommons.org/licenses/by-nc/3.0) which permits unrestricted noncommercial use, distribution, and reproduction in any medium, provided the original work is properly cited.

Copyright () 2012 by Korean Academy of Rehabilitation Medicine

\section{INTRODUCTION}

Cerebral palsy is a developmental disability with symptoms of restriction on motion, posture, and activities due to non-developmental physical changes followed by diseases that occurin the brain of children who are physically growing. ${ }^{1}$ Cerebral palsy disables the control of contraction and relaxation of muscles. This makes the ac- 
tivities of daily living difficult, resulting in the exertion of greater physical strength for any movement compared to normal people. In particular, patients with cerebral palsy of hemiplegia or quadriplegia experience disorders of the upper limb, and experience difficulties in using their upper limbs routinely.

Patients with cerebral palsy need steady upper limb training because cerebral palsy may lead to muscle contracture and functional deterioration. Treatments for enhancing upper limb function are based on a range of motion exercise, ${ }^{2}$ neurodevelopment, oral medication including baclofen, and injection such as botox injection. ${ }^{3}$ As for other treatment, constraint-induced movement therapy (CIMT) ${ }^{4}$ that repeats functional motions using specific and an approach method of exercise learning are currently utilized. Considering that a weakness of muscular strengths of children with cerebral palsy is one of the important reasons influencing motorfunction, enhancing muscular strength is a major treatment for motor performance. ${ }^{5}$ According to one study, it has been reported that training to intensify muscular strength especially on the lower limbs is effective in enhancing walking function. ${ }^{6}$ Lee reported that comprehensive hand repetitive intensive strength trainingis effective in enhancing the function of the upper limbs and muscular strength.

Butler has reported that experience-oriented proof of verifying treatment with introduction of neurology enhanced function of the upper limb is insufficient. ${ }^{8}$ Chang and others have reported the comparison of the speed of motion, criteria of physical strength, and normalize jerk score between normal children and children with cerebral palsy. ${ }^{9}$ However, there are only a few studies onenhancement of upper limb functions in children with cerebral palsy through active physical training of the upper limbs. In addition, there are no trials that have objectively suggested the result through motion analysis. This study is intended to apply comprehensive hand repetitive intensive strengthtraining (CHRIST) objectively and quantitatively, proposing its effects through motion analysis.

\section{MATERIALS AND METHODS}

\section{Study Subjects}

With cooperation from the hospital and social welfare center, children diagnosed with cerebral palsy were re- cruited for this. The ages of children with hemiplegia and quadriplegia among cerebral palsy were restricted to between 6 and 13, targeting those with normal communication skills and cognitive functions. However, children with a history of orthopedic surgery or any other operations held 6 months prior to conducting the study, or with damage of the musculoskeletal system including fracture of upper limb and contracture, or having undergone administration of medicine influencing muscular strength and spasticity were excluded from this study.

A total of 19 children were recruited finally, including 10 boys and 9 girls. The average age was 8.8 years. The average height was $125.3 \mathrm{~cm}$, and the average weight was $24.4 \mathrm{~kg}$. Ten of them suffered from hemiplegia, whereas the other 9 children had quadriplegia. Using a table of random numbers, the children were randomly divided into two groups. There were a total of 5 boys and 5 girls in the experimental group with an average age of 8.6 years. Their average height was $123.8 \mathrm{~cm}$, and average weight was $24.2 \mathrm{~kg}$. Five had hemiplegia, whereas the other five had quadriplegia. There were a total of five boys and four girls in the control group with an average age of 9.1. Their average height was $127.0 \mathrm{~cm}$, and average weight was 24.4 $\mathrm{kg}$. Five of them had hemiplegia, whereas the other five had quadriplegia. There was no statistically significant difference between the characteristics of the study subjects for the two groups ( $p>0.05)$ (Table 1).

Table 1. Characteristics of Subjects in This Study

\begin{tabular}{lccc}
\hline & $\begin{array}{c}\text { Experimental } \\
\text { group } \\
(\mathbf{n}=\mathbf{1 0})\end{array}$ & $\begin{array}{c}\text { Control } \\
\text { group } \\
(\mathbf{n}=\mathbf{9})\end{array}$ & p-value \\
\hline Sex & 5 & & 0.842 \\
$\quad$ Male & 5 & 5 & \\
$\quad$ Female & & 4 & \\
Mean age (years) & $8.6 \pm 1.9$ & $9.1 \pm 1.8$ & 0.604 \\
Cerebral palsy type & & & 0.842 \\
$\quad$ Hemipelgia & 5 & 5 & \\
$\quad$ Quadriplegia & 5 & 4 & \\
Height & $123.8 \pm 10.9$ & $127.0 \pm 13.2$ & 0.661 \\
Weight & $24.2 \pm 7.0$ & $24.2 \pm 7.2$ & 0.968 \\
MACS & $2.8 \pm 0.9$ & $2.1 \pm 0.6$ & 0.156 \\
\hline
\end{tabular}

Data presented as mean \pm standard deviation MACS: Manual ability classification system 


\section{Study method}

Ten children classified into the experimental group had comprehensive hand repetitive intensive strength training for 60 minutes per session, three sessions a week, at a total of 30 sessions per ten weeks. Comprehensive hand repetitive intensive strength training included treadmilltraining for the upper limb with body weight supporting the using upper limb. The posture of an arm swing was to maintain the joints of the elbows and wrist as much as possible, and the shoulder joint was allowed to be flexed by 160 degrees. The posture of the arm stance supported

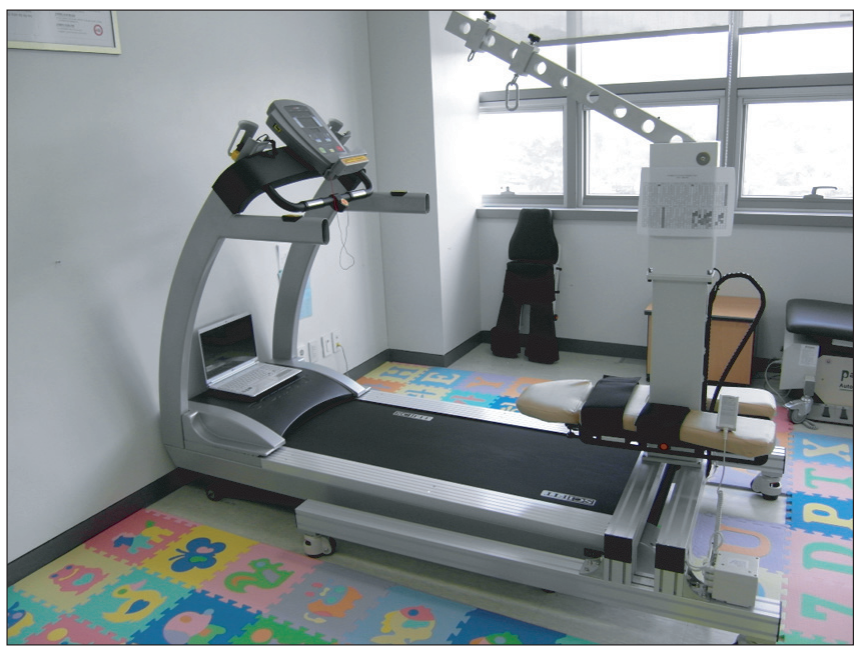

Fig. 1. The Comprehensive Hand Repetitive Intensive Strengthening Training (CHRIST) System. the weight using metacarpal heads, hands, and elbows, and the shoulder joints were allowed to be flexed by 90 degrees. By maintaining the posture of the quadruped by repeating this posture, the abdomen and weight of the lower limb were supported using a support board under the abdomen (Fig. 1, 2). In order to maintain posture, the therapist observed the study subject next to them, helping them maintain the correct posture if needed. They showed videos in which children linkedto watch or play music that they could concentrate on the training for 60

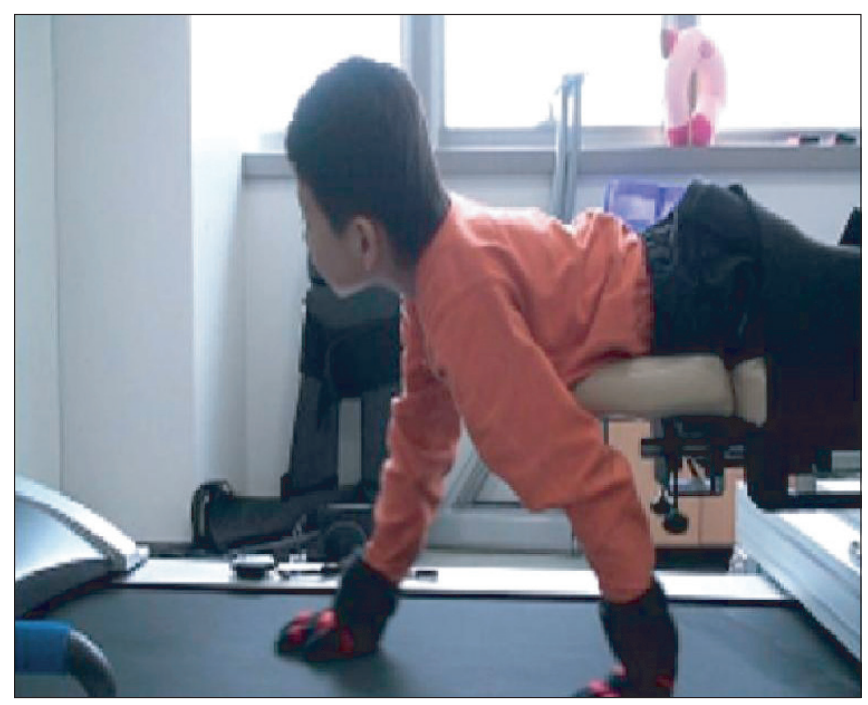

Fig. 2. The Comprehensive Hand Repetitive Intensive Strengthening Training (Quadruped posture).
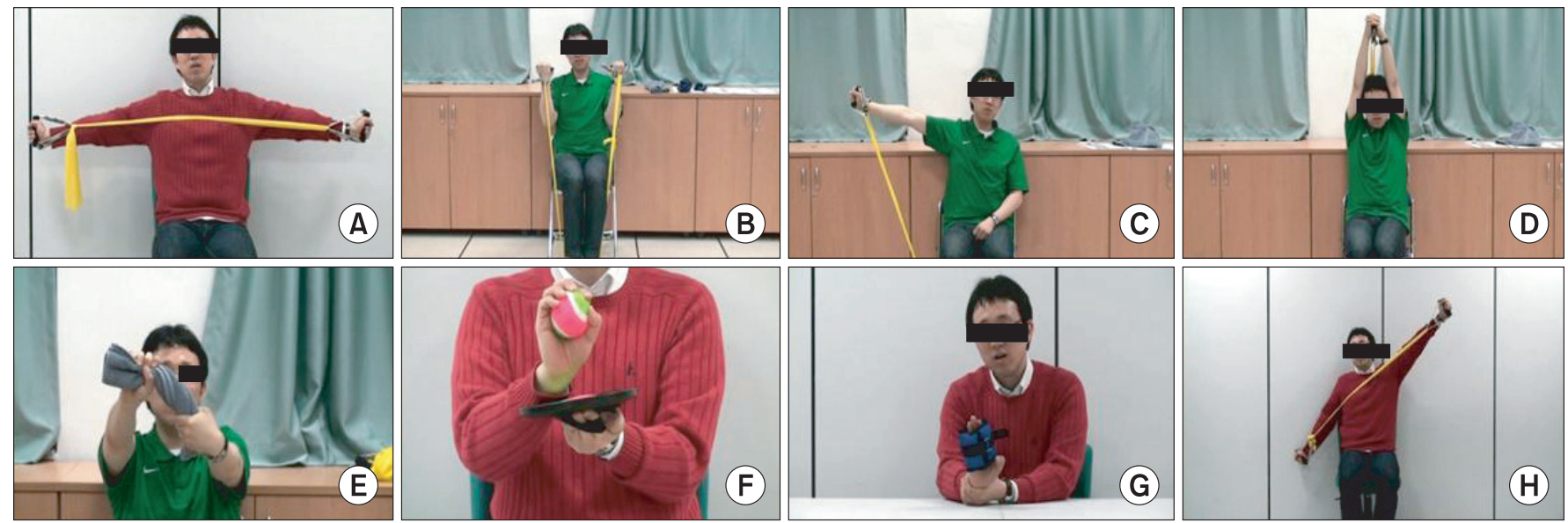

Fig. 3. Home Exercise Program. (A) Shoulder extension muscle strengthening with theraband. (B) Elbow flexion muscle strengthening with theraband. (C) Shoulder abductionmuscle strengthening with theraband. (D) Elbow extension muscle strengthening with theraband. (E) Hand grip, forearm flexion and extension muscle strengthening with towel. (F) Hand grip and wrist extension muscle strengthening with velcro board. (G) Wrist extension muscle strengthening with sandbag. (H) Shoulder abduction-extension muscle strengthening with theraband. 
minutes without a break time. The speed of the treadmill for the upper limbs was $0.3-1.2 \mathrm{~km} / \mathrm{h}$.

Five children classified into the control group were given instructions for 60 minutes per a session, three sessions a week, at a total of 30 sessions per 10 weeks by creating a home program, which consisted of a total of 8 exercises that enhanced the muscular strength of the upper limb including thetriceps brachii and extensor carpi radialis, and conveyed an effect of reaching the muscles out.

This exercise consisted of shoulder extension muscle, elbow flexion muscle, shoulder abduction muscle, and elbow extension muscle strengthening with a theraband, as well as hand grip, forearm flexion and extension muscle strengthening with towel, hand grip and wrist extension muscle strengthening with a velcro board, wrist extension muscle strengthening with sandbag and shoulder abduction-extension muscle strengthening with a theraband (Fig. 3).

Motion analysis of the upper limbs was evaluated by using an 8-camera (VICON, Oxford, UK). The estimation method reached out to the upper limb after attaching 24 VICON NEXUS upper limb model on the upper limb of children with cerebral palsy before and after a total of 30 sessions of training to analyze the motion (Fig. 4). Each child was seated on the chair and desk, which were adjusted according to the height of the children with cerebral palsy, and the posture of the upper limb was vertically maintained with the ground, with 90 degrees elbows flexion so that the palms were maintained parallel to the ground. In addition, the wrists were maintained in a natural posture so that the line of wrist articulations was

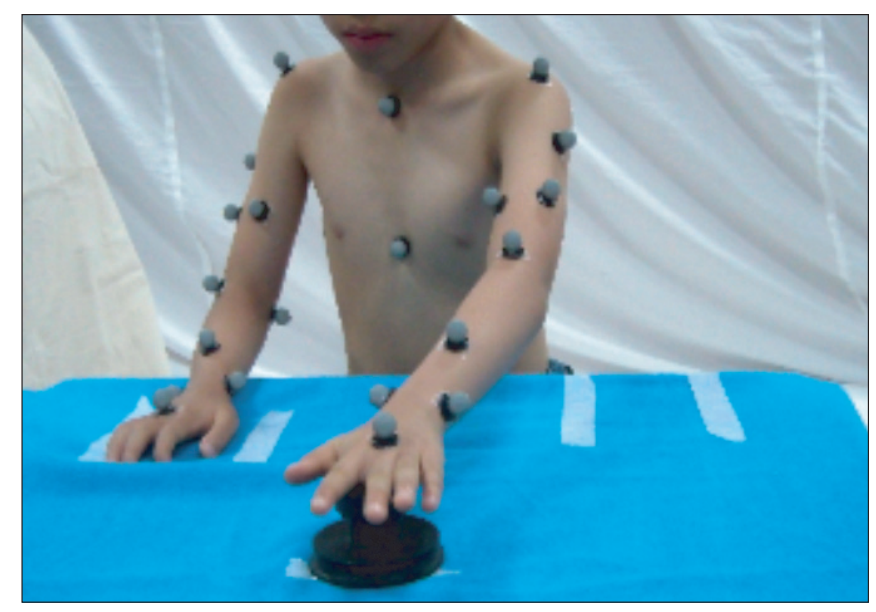

Fig. 4. The experimental setup of the motion analysis. consistent with the edge of the desk. Maintaining this posture, the left and right upper limbs were reached out five times with a convenient speed and fast speed to perform the motion analysis. During this procedure, movement time, mean velocity, normalized jerk score, mean angular velocity of shoulder, elbow, and wrist at a comfortable speed and at fast speed were estimated, comparing the experimental group and control group for mean angular velocity for each shoulder, elbow and wrist.

Movement time (MT) reflects the speed of reaching out, and mean velocity and mean angular velocity (MAV) of each articulation reflect the degree of strength of reaching out. This is a value of maximum average velocity when the angle of each articulation becomes widened or narrowed when reaching out, which indicates torque power of articulation. Normalized jerk score (NJS) ${ }^{9,10}$ indicates smoothness of reaching out, using thetime and distance that VICON NEXUS upper limb model has moved via routes in three dimensional space, indicating the nature of behaviors. As the value becomes smaller, the movement of VICON NEXUSupper limb model is more natural and not shaking.

\section{Statistical analysis}

A Wilcoxon test was conducted to identify differences between before and after treatment for both groups. In order to identify differences between the two groups, a Mann-Whitney test was conducted on the changed amount to identify the difference between the two groups.

\section{RESULTS}

In the comparison of motion analysis before and after conducting training, the experimental group showed a significant difference in the movement time of comfortable speed and fast speed, mean velocity, normalizedjerk score, mean angular velocity of wrist, shoulder, elbows and normalized jerk score of wrist on reaching motion $(p<0.05)$ (Table 2). Control group showed a significant difference only for mean velocity of comfortable speed on reaching motion $(\mathrm{p}<0.05)$ (Table 3$)$. Both the experimental and control group were estimated with mean velocity and mean angular velocity of shoulders, wrists, and wrist faster at the fast speed than in the comfortable speed.

In the comparison of motion between experimental 
and control groups, there was a significant difference in the movement time, angular velocity of wrist of comfortable speed and normalizedjerk score of elbow at fast speed $(\mathrm{p}<0.05)$ (Table 4).

\section{DISCUSSION}

The Comprehensive Hand Repetitive Intensive Strength Training (CHRIST) program is designed by Lee et al., and provides special training for enhancing muscular strength of the upper limp using a treadmill. In this study, the treadmill device designed by Lee et al. was modified to support the weight of the study subjects in a more stable way and was adjusted according to the physical condition (Fig. 1). Muscle strengthening training is known for a specific training to children with cerebral palsy without an increase of spasticity and other side effects. ${ }^{5,6}$ In addition, among many other methods of estimating the capability of exercising, motion analysis has the advantage of quantitatively estimating and comparing kinematic vari-

Table 2. Comparison of Kinematic Variables between Pre- and Post-training in the Experimental Group

\begin{tabular}{|c|c|c|c|c|c|c|}
\hline & \multicolumn{3}{|c|}{ Comfortable speed } & \multicolumn{3}{|c|}{ Fast speed } \\
\hline & Pre & Post & p-value & Pre & Post & p-value \\
\hline MT & $3.3 \pm 1.9$ & $2.2 \pm 0.6$ & $0.005^{*}$ & $2.2 \pm 1.4$ & $1.6 \pm 0.6$ & $0.005^{*}$ \\
\hline MV & $37.9 \pm 16.2$ & $59.4 \pm 29.2$ & $0.005^{*}$ & $60.3 \pm 23.3$ & $93.4 \pm 47.5$ & $0.005^{*}$ \\
\hline NJS & $376.2 \pm 226.7$ & $207.9 \pm 122.4$ & $0.013^{*}$ & $439.7 \pm 290.9$ & $240.3 \pm 108.6$ & $0.007^{*}$ \\
\hline SMAV & $76.4 \pm 36.6$ & $119.1 \pm 67.2$ & $0.007^{*}$ & $108.7 \pm 48.3$ & $173.2 \pm 97.7$ & $0.009^{*}$ \\
\hline EMAV & $61.4 \pm 36.6$ & $84.1 \pm 38.8$ & $0.005^{*}$ & $93.4 \pm 43.9$ & $126.1 \pm 57.2$ & $0.013^{*}$ \\
\hline WMAV & $45.5 \pm 19.1$ & $67.3 \pm 28.0$ & $0.005^{*}$ & $69.1 \pm 36.0$ & $107.9 \pm 60.5$ & $0.005^{*}$ \\
\hline S_NJS & $391.7 \pm 230.6$ & $284.4 \pm 215.2$ & 0.285 & $427.2 \pm 240.6$ & $299.4 \pm 160.9$ & 0.093 \\
\hline E_NJS & $1,188.5 \pm 164.1$ & $737.2 \pm 355.2$ & $0.017^{*}$ & $1,362.7 \pm 979.2$ & $692.9 \pm 236.9$ & $0.022^{*}$ \\
\hline W_NJS & $2,081.8 \pm 918.5$ & $1,448.5 \pm 534.8$ & $0.009^{*}$ & $2,187.2 \pm 1,364.0$ & $1,557.2 \pm 740.2$ & $0.007^{*}$ \\
\hline
\end{tabular}

Analyzed by Wilcoxon signed rank test. Data are presented as mean \pm standard deviation

MT: Movement time (second), MV: Mean velocity (cm/s), NJS: Normalized jerk score, SMAV: Shoulder mean angular velocity (cm/s), EMAV: Elbow mean angular velocity $(\mathrm{cm} / \mathrm{s})$, WMAV: Wrist mean angular velocity (cm/s), S_NJS: Shoulder normalized jerk score, E_NJS: Elbow normalized jerk score, W_NJS : Wrist normalized jerk score

Significant difference between pre and post $\left({ }^{*} \mathrm{p}<0.05\right)$

Table 3. Comparison of Kinematic Variables between Pre- and Post-training in the Control Group

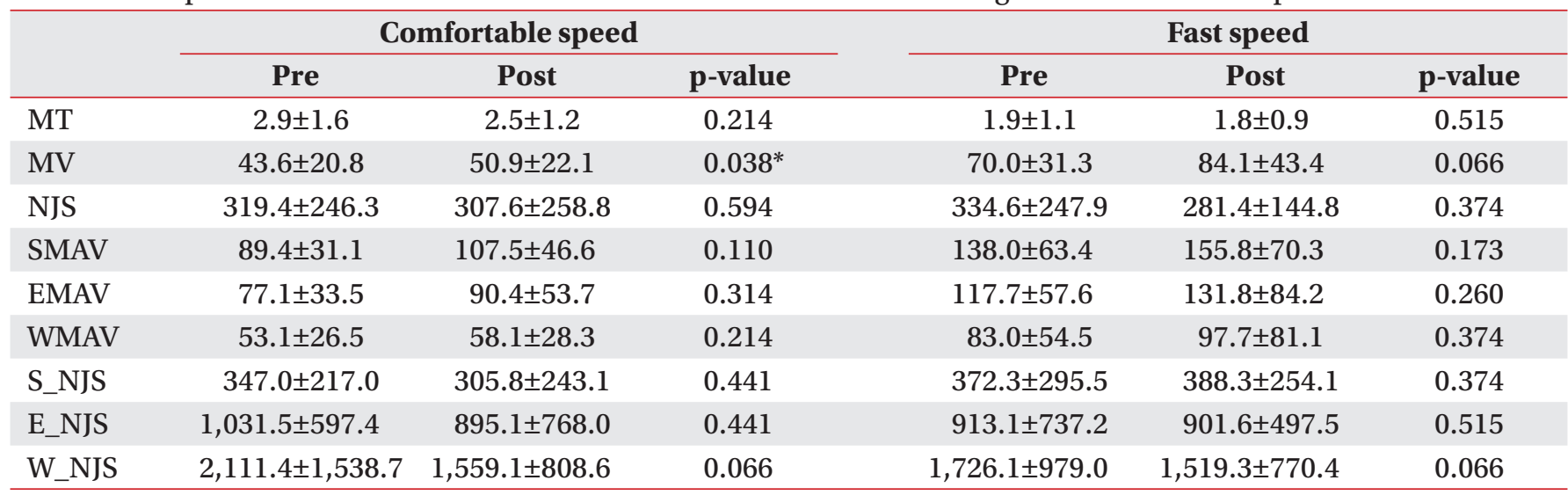

Analyzed by Wilcoxon signed rank test. Data are presented as mean \pm standard deviation.

MT: Movement time (second), MV: Mean velocity (cm/s), NJS: Normalized jerk score, SMAV: Shoulder mean angular velocity (cm/s), EMAV: Elbow mean angular velocity $(\mathrm{cm} / \mathrm{s})$, WMAV: Wrist mean angular velocity (cm/s), S_NJS: Shoulder normalized jerk score, E_NJS : Elbow normalized jerk score, W_NJS : Wrist normalized jerk score Significant difference between pre and post $\left({ }^{*} \mathrm{p}<0.05\right)$ 
Table 4. Difference of Pre- and Post-training Test between the Control and Experimental Group

\begin{tabular}{|c|c|c|c|c|c|c|}
\hline & \multicolumn{3}{|c|}{ Comfortable speed } & \multicolumn{3}{|c|}{ Fast speed } \\
\hline & Control & Experimental & p-value & Control & Experimental & p-value \\
\hline MT & $-0.4 \pm 1.0$ & $-1.1 \pm 1.5$ & $0.043^{*}$ & $-0.1 \pm 0.4$ & $-0.6 \pm 0.9$ & 0.113 \\
\hline MV & $7.4 \pm 8.2$ & $21.5 \pm 23.0$ & 0.211 & $14.1 \pm 18.4$ & $33.1 \pm 31.9$ & 0.211 \\
\hline NJS & $-11.8 \pm 93.0$ & $-168.3 \pm 199.4$ & 0.065 & $-53.2 \pm 166.3$ & $-199.4 \pm 260.2$ & 0.156 \\
\hline SMAV & $18.0 \pm 34.0$ & $42.7 \pm 55.9$ & 0.356 & $17.8 \pm 38.3$ & $64.5 \pm 71.1$ & 0.243 \\
\hline EMAV & $13.3 \pm 36.9$ & $22.7 \pm 24.8$ & 0.549 & $14.2 \pm 38.0$ & $32.7 \pm 31.9$ & 0.315 \\
\hline WMAV & $5.1 \pm 15.1$ & $21.8 \pm 15.8$ & $0.035^{*}$ & $14.6 \pm 38.5$ & $38.8 \pm 38.9$ & 0.156 \\
\hline S_NJS & $-50.8 \pm 194.5$ & $-107.3 \pm 281.4$ & 0.604 & $16.0 \pm 128.3$ & $-127.8 \pm 256.3$ & 0.113 \\
\hline E_NJS & $-136.4 \pm 596.9$ & $-451.3 \pm 472.3$ & 0.182 & $-11.5 \pm 375.8$ & $-669.8 \pm 994.6$ & $0.043^{*}$ \\
\hline W_NJS & $-552.3 \pm 880.1$ & $-633.3 \pm 592.9$ & 0.497 & $-206.8 \pm 266.1$ & $-630.0 \pm 670.4$ & 0.113 \\
\hline
\end{tabular}

Values of control and experimental groups mean arithmetical difference (values from post-training minus those from pre-training) from the figures of Table 2 and 3. Analyzed by Mann-Whitney test. Data are presented as mean \pm standard deviation

MT: Movement time (second), MV: Mean velocity (cm/s), NJS: Normalized jerk score, SMAV: Shoulder mean angular velocity (cm/s), EMAV: Elbow mean angular velocity $(\mathrm{cm} / \mathrm{s})$, WMAV: Wrist mean angular velocity (cm/s), S_NJS: Shoulder normalized jerk score, E_NJS: Elbow normalized jerk score, W_NJS: Wrist normalized jerk score Significant difference between experimental group and control group $\left({ }^{*} \mathrm{p}<0.05\right)$

ables, such as movement time and normalized jerk score of each articulation. ${ }^{9}$

This study was intended to identify how comprehensive hand repetitive intensive strength training influences the reaching motion on activities of daily living relating to the upper limbs of children with cerebral palsy through motion analysis. In general, reaching out an upper limb actively determines the location of hands, and moving the hands near that location to interact with the surrounding environment. Such motion is a basic but important motion of the upper limb as an activity of daily living including eating, opening or closing the door, or pressing a switch or operation button. Children with cerebral palsy are prone to experience difficulties in reaching upper limb out due to spasticity. Therefore, an evaluation of reachingout effectively determines changes of upper limbs after rehabilitation. ${ }^{11}$ This is because an analysis of reaching out motion conveys an objective knowledge as to movement velocity, criterion of the strength, and normalized jerk score of the upper limb. ${ }^{12,13}$

This study conducted comprehensive hand repetitive intensive strength training for the experimental group, and used the home program for the control group to evaluate kinematic variables of the two groups and compared differences before and after for these two groups. A comfortable speed selected by the study subject was separately applied with the motion of moving with a speed as fast as possible for evaluation. Here, the reaching out motion on activities of daily living reflected naturalness and functional ability of the upper limb. However, a reaching motion at the fast speed reflects the maximum allowed torque power of articulation and a degree of strength in upper limb function. Smoothness of the movement can be utilized as a good index to measure a degree of spasticity for normalized jerk score of the motion. Normalized jerk score ${ }^{9,10}$ indicates smoothness of the reaching out motion, derived via a formula of

$$
\mathrm{NJS}=\sqrt{\frac{1}{2} \times \int\left(\mathrm{\gamma}^{\prime \prime \prime}\right)^{2} d t \times\left(\frac{\mathrm{t}^{5}}{l^{2}}\right)}
$$

where $\gamma^{\prime \prime \prime}$ of the formula is a third time derivative of position data, a value differentiated three times for the location data on the time that indicates a jerk of acceleration of unit time. $t$ is a movement time, while $l$ indicates the amplitude. In this study, it was confirmed that the normalized jerk score at the comfortable speed was lower than the one at the fast speed. This indicates that the motion of reaching upper limb out is more natural at the comfortable speed than at the fast speed, showing that spasticityincreased at the fast velocity. On the opposite, mean time and mean angular velocity of shoulders and wrists were estimatedfaster than those at comfortable speed. The degree of strength at fast speed and torque power of articulation increased more at speed velocity than fast speed. 
From the result of motion analysis of before and after in each group, the experimental group showed a significant difference in the movement time of comfortable speed and fast speed, mean velocity, normalized jerk score, mean angular velocity of wrist, shoulder, elbows and normal jerk score of wrist on reaching motion $(\mathrm{p}<0.05)$. However, there was no significant difference in the normalized jerk score of shoulder while moving ( $p>0.05)$. This reflects a training characteristic that puts priority on the elbow and wrist. In addition, derivation of proper posture and intensification of muscular strength of wrists through comprehensive hand repetitive intensive strength training can be be effective on the movement time for the upper limb, torque power of the wrist, and normalized jerk score of the wrist while moving. The group that underwent the home program showed a significant difference only on the mean movement time at comfortable velocity $(\mathrm{p}<0.05)$. Such a result suggests that the home program improves muscular strength, influencing movement velocity, but did not influence the angular velocity of articulation and normalized jerk score.

The difference between the experimental and control group showed a significant difference since a reaching motion improved only for movement time at comfortable speed, angular velocity of wrist, and normalized jerk score of wrist at fast speed $(\mathrm{p}<0.05)$.

There were a few limitations in this study. First, the study was conducted only using the motion of reaching out of the upper limb. Among motions involved in activities of daily living, many other motions including bringing hands to mouth or moving hands over the heads were excluded but only considered a motion of forward in order to touch for this study. There should be an objective and quantitative study on the motion analysis via various motions occurred on activities of daily living. Second, a difference of electrical activation for the muscle of the upper limb could not be analyzed. When analyzing the muscle electrical activation while reaching out, detailed changes of each muscle can be understood, and may be available to suggest correlations of movement and muscle activation from comprehensive hand repetitive intensive strength training. Third, tools specially designed for comprehensive hand repetitive intensive strength training were definitely helpful for training of the upper limbs of children with cerebral palsy, but at least one therapist was required to correct posture during training, taking
60 minutes of training time, which requires tremendous efforts by the therapist. Tools specially designed for the training had to be modified in order to conduct effective treatment in the future.

\section{CONCLUSION}

In this study, a total of 19 children with cerebral palsy were selectedto undergo comprehensive hand repetitive intensive strength training and general rehabilitation 30 times in ten weeks on ten children in the experimental group. However, nine children in the control group were given home-program and general rehabilitation, comparing the reaching motion at a comfortable speed and at a fast speed through motion analysis. The difference between before and after treatment of each group was compared. The group that received comprehensive hand repetitive intensive strength training showed a significant difference for the motion of reaching hands out both at comfortable speed and at fast speed since movement time, mean velocity, and normalized jerk score of upper limb were enhanced after having a training. This objectively confirms that comprehensive hand repetitive intensive strength training is a treatment that is helpful toenhance upper limb movement of the elbow and wrist. This highly suggests utilizing comprehensive hand repetitive intensive strength training as a rehabilitation treatment that enhances capability of exercising for upper limbsof children with cerebral palsy.

\section{ACKNOWLEDGEMENTS}

This research was supported by a grant (10-B-03) by Korea National Rehabilitation Research Institute.

\section{REFERENCES}

1. Bax M, Goldstein M, Rosenbaum P, Leviton A, Paneth N, Dan B, Jacobsson B, Damiano D. Proposed definition and classification of cerebral palsy, April 2005. Dev Med Child Neurol 2005; 47: 571-576

2. Ketelaar M, Vermeer A, Hart H, van Petegem-van Beek E, Helders PJ. Effects of a functional therapy program on motor abilities of children with cerebral palsy. Phys Ther 2001; 81: 1534-1545

3. Boyd RN, Morris ME, Graham HK. Management of 
upper limb dysfunction in children with cerebral palsy: a systematic review. Eur J Neurol 2001; 8 Suppl 5: 150-166

4. Taub E, Ramey SL, DeLuca S, Echols K. Efficacy of constraint-induced movement therapy for children with cerebral palsy with asymmetric motor impairment. Pediatrics 2004; 113: 305-312

5. Damiano DL, Dodd K, Taylor NF. Should we be testing and training muscle strength in cerebral palsy? Dev Med Child Neurol 2002; 44: 68-72

6. Dodd KJ, Taylor NF, Damiano DL. A systematic review of the effectiveness of strength-training programs for people with cerebral palsy. Arch Phys Med Rehabil 2002; 83: 1157-1164

7. Lee DR, You JH, Lee NG, Oh JH, Cha YJ. Comprehensive Hand Repetitive Intensive Strengthening Training (CHRIST)-induced morphological changes in muscle size and associated motor improvement in a child with cerebral palsy: an experimenter-blind study. NeuroRehabilitation 2009; 24: 109-117
8. Butler C, Darrah J. Effects of neurodevelopmental treatment (NDT) for cerebral palsy: an AACPDM evidence report. Dev Med Child Neurol 2001; 43: 778-790

9. Chang JJ, Wu TI, Wu WL, Su FC. Kinematical measure for spastic reaching in children with cerebral palsy. Clin Biomech (Bristol, Avon) 2005; 20: 381-388

10. Teulings HL, Contreras-Vidal JL, Stelmach GE, Adler $\mathrm{CH}$. Parkinsonism reduces coordination of fingers, wrist, and arm in fine motor control. Exp Neurol 1997; 146: 159-170

11. McCrea PH, Eng JJ, Hodgson AJ. Biomechanics of reaching: clinical implications for individuals with acquired brain injury. Disabil Rehabil 2002; 24: 534-541

12. Reid S, Elliott C, Alderson J, Lloyd D, Elliott B. Repeatability of upper limb kinematics for children with and without cerebral palsy. Gait Posture 2010; 32: 10-17

13. Mackey AH, Miller F, Walt SE, Waugh MC, Stott NS. Use of three-dimensional kinematic analysis following upper limb botulinum toxin A for children with hemiplegia. Eur J Neurol 2008; 15: 1191-1198 